\title{
NLRP3 Agonist BMS-986299
}

National Cancer Institute

\section{Source}

National Cancer Institute. NLRP3 Agonist BMS-986299. NCI Thesaurus. Code C155945.

A nucleotide-binding domain and leucine-rich repeat (NLR) family pyrin domain containing 3 (NLRP3; NACHT, LRR and PYD Containing Protein 3; NALP3) agonist with potential immunomodulatory and antineoplastic activities. Upon administration, NLRP3 agonist BMS-986299 binds to and activates NLRP3, potentially promoting NLRP3 inflammasome-mediated secretion of interleukin-8 (IL-8), which may induce tumoricidal activity of natural killer (NK) cells against tumor cells. NLRP3, a sensor component of the NLRP3 inflammasome plays a significant role in immunity and inflammation, and may protect against tumorigenesis in some cancers. 